\title{
uPAR signaling is under par for the podocyte course
}

\author{
Friedrich C. Luft
}

Published online: 17 November 2012

(C) Springer-Verlag Berlin Heidelberg 2012

The urokinase receptor, also known as uPAR or cluster of differentiation (CD) 87 , is a multidomain glycoprotein tethered to the cell membrane with a glycosylphosphatidylinositol anchor. UPAR was originally identified as the binding site for the extracellular protease urokinase-type plasminogen activator (UPA) on the cell surface. However, uPAR interacts with many other proteins such as vitronectin, the uPAR-associated protein, and integrins [1]. UPAR is a part of the plasminogen activation system, which is not only involved in hemostasis but also in tissue reorganization including mammary gland involution and wound healing. The plasminogen activation system is important in reorganizing tissues through proteolysis. uPAR restricts plasminogen activation to the immediate vicinity of the cell membrane. Coordination of extracellular matrix (ECM) proteolysis and cell signaling underlies uPAR's important function in cell migration, proliferation, and survival. As a result, various pharmaceutical strategies are under development to target UPAR in combating cancer and other conditions [2].

uPAR signaling has also been implicated in cardiovascular disease [3]. The process of atherosclerosis involves the recruitment of vascular smooth muscle cells (VSMC) from the media into the intima. VSMC subsequently proliferate and form a neointima in a process called vascular remodeling. In this process, the VSMC express high levels of uPA and UPAR. uPA mediates ECM degradation and is important to cell adhesion, migration, and proliferation during the remodeling process. The uPA/uPAR complex also conducts intracellular signaling. For instance, Kiyan et al. recently identified a new molecular mechanism controlling VSMC

\section{F. C. Luft $(\bowtie)$}

Experimental and Clinical Research Center,

Max-Delbrück Center and Charité Medical Faculty,

Lindenbergerweg 80 ,

13125, Berlin, Germany

e-mail: luft@charite.de phenotypic modulation in vitro and in vivo. They found that uPAR acts together with myocardin, the platelet-derived growth factor- $\beta$ receptor, and SUMOylated RanGAP1 to mediate this process [4].

uPAR signaling is also important to kidney disease. UPAR is expressed by several cell types in diseased kidneys. Studies in uPAR gene-deleted mice suggest that uPAR serves a protective role in the process of renal fibrosis. For instance, UPAR interacts with specific co-receptors to degrade plasminogen activator inhibitor 1 and ECM. Moreover, a soluble short form of UPAR (suPAR) exists which has leukocyte chemo-attractant properties [5]. suPAR is the result of cleavage and release of membrane-bound uPAR.

The relevance of uPAR to renal disease gained an additional dimension when Wei et al. [6] reported that uPAR signaling in glomerular podocytes leads to foot process effacement and proteinuria. Podocytes regulate the glomerular filtration barrier. We now know that focal segmental glomerulosclerosis (FSGS), a major cause of end-stage renal disease worldwide, is a podocyte disease. Wei et al. [6] found that mice lacking UPAR were protected from lipopolysaccharide (LPS)-mediated proteinuria; however, they developed disease after expression of a constitutively active $\beta 3$ integrin. Wei et al. then performed gene transfer studies to show that uPAR expression in podocytes was required for development of LPS-mediated proteinuria, a commonly utilized model. The investigators then showed that uPAR was required to activate $\alpha \mathrm{v} \beta 3$ integrin in podocytes, promoting cell motility and activation of small GTPases. Blockade of $\alpha v \beta 3$ integrin reduced podocyte motility in vitro and lowered proteinuria. These findings introduced uPAR signaling to the arena of glomerular permeability.

Wei et al. [7] then introduced the next startling findings regarding UPAR in podocytes. They reported that suPAR, introduced above, is elevated in about two thirds of patients with FSGS, but not in patients with other glomerular 
diseases. FSGS commonly recurs after transplantation. Wei et al. [7] also found that high concentrations of suPAR before transplantation were associated with increased risk for recurrence of FSGS after transplantation. They next used three mouse models to explore the effects of suPAR on kidney function and morphology. They showed that circulating suPAR activated podocyte $\beta 3$ integrin, causing foot process effacement, proteinuria, and a histological picture consistent with FSGS. The findings suggest that FSGS could develop when suPAR is sufficient to activate the podocyte $\beta 3$ integrin. Conceivably, suPAR could be a therapeutic target in FSGS.

Clinicians commonly treat FSGS with immunosuppressive drugs and sometimes patients respond favorably, although the mechanisms involved were completely unclear. Corticosteroids, cyclophosphamide, and calcineurin inhibitors such as cyclosporine, are commonly employed. Calcineurin inhibitors interfere with nuclear factor of activated $\mathrm{T}$ cell (NFAT) signaling. Calcineurin is a phosphatase that dephosphorylates NFATc, which moves to the nucleus of $\mathrm{T}$ cells and increases the activity of genes coding for IL-2 and related cytokines. Ciclosporin prevents the dephosphorylation of NFATc by binding to cyclophilin. However, podocytes are also equipped with NFAT. In this issue of $J \mathrm{Mol}$ Med, Zhang and other members of the team involved in the above two studies [8] show that inducible podocyte-specific expression of constitutively active NFATc1 increases podocyte uPAR expression by binding to the Plaur gene promoter, as they show in chromatin immunoprecipitation assays. The Plaur gene encodes uPAR. Zhang et al. [8] show that pathological uPAR signals in podocytes are independent of the $\mathrm{T}$ cell effects mentioned above. uPAR signaling in podocytes affects cell motility via activation of the $\beta 3$ integrin. Cyclosporin, NFAT-siRNA, or the cell-permeable NFAT inhibitor (11R-VIVIT) can all block this activation, at least in mouse models of glomerular disease. The team makes strong arguments implicating podocyte uPAR as a downstream target of NFAT. By so doing, they provide further fascinating insights into the pathogenesis of FSGS. A loose schematic is presented in Fig. 1.

The therapeutic relevance of the uPAR-FSGS story does not end with cyclosporine, which is after all, pretty toxic stuff. Amiloride has a significant role in the reduction of podocyte cell motility in vitro and proteinuria in mice. Zhang et al. [9] have also shown that amiloride inhibits the induction of the Plaur gene and UPAR mRNA, thereby reducing uPAR-mediated $\beta 3$ integrin activation in LPStreated podocytes. Interestingly, the off-target effect of amiloride was independent of its function as epithelial sodium channel blocker and different from the actions of triamterene. Amiloride was also effective in the LPS mouse model of transient proteinuria and in the five of six nephrectomy rat
Fig. 1 uPAR signals through $\beta 3$ integrins to stimulate kidney podocytes. Vitronectin (in the ECM), which binds uPAR and $\beta 3$ integrins, is required for the activity of this pathway. Urokinase does not seem to have an important role in UPAR signaling through $\beta 3$ integrin but does influence uPAR cleavage. Src kinases are activated by the UPAR $-\beta 3$ integrin interaction. Rho family GTPases are activated. NFAT signaling is constitutive and activates the gene encoding uPAR (adapted in part from [1])

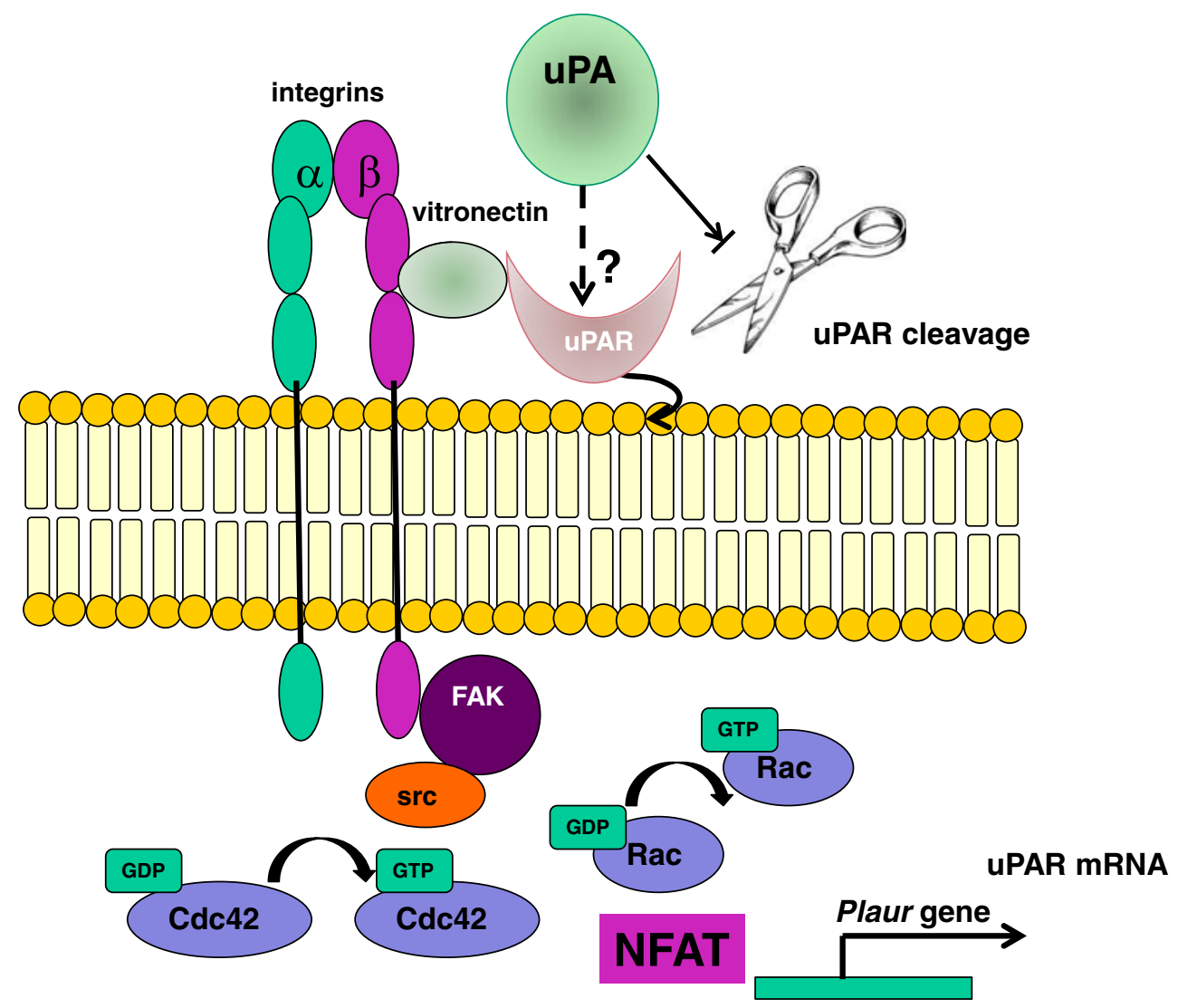


FSGS model. The mechanisms involved a significant inhibition of podocyte UPAR induction. In addition, amiloride attenuated glomerulosclerosis histologically. Thus, the exciting question can be raised that amiloride could ameliorate a pathological uPAR- $\beta 3$ integrin signaling axis in FSGS patients.

Respectfully,

Friedrich C. Luft

\section{References}

1. Smith HW, Marshall CJ (2010) Regulation of cell signalling by uPAR. Nat Rev Mol Cell Biol 11:23-36

2. Mekkawy AH, Morris DL, Pourgholami MH (2009) Urokinase plasminogen activator system as a potential target for cancer therapy. Future Oncol 5:1487-1499

3. Fuhrman B (2012) The urokinase system in the pathogenesis of atherosclerosis. Atherosclerosis 222:8-14
4. Kiyan Y, Limbourg A, Kiyan R, Tkachuk S, Limbourg FP, Ovsianikov A, Chichkov BN, Haller H, Dumler I (2011) Urokinase receptor associates with myocardin to control vascular smooth muscle cells phenotype in vascular disease. Arterioscler Thromb Vasc Biol 32:110-122

5. Eddy AA (2009) Serine proteases, inhibitors and receptors in renal fibrosis. Thromb Haemost 101:656-664

6. Wei C, Möller CC, Altintas MM, Li J, Schwarz K, Zacchigna S, Xie L, Henger A, Schmid H, Rastaldi MP et al (2008) Modification of kidney barrier function by the urokinase receptor. Nat Med 14:5563

7. Wei C, El Hindi S, Li J, Fornoni A, Goes N, Sageshima J, Maiguel D, Karumanchi SA, Yap HK, Saleem M et al (2011) Circulating urokinase receptor as a cause of focal segmental glomerulosclerosis. Nat Med 17:952-960

8. Zhang B, Shi W, Ma J, Sloan A, Faul C, Wei C, Reiser J, Yang Y, Liu S, Wang W (2012) The calcineurin-NFAT pathway allows for urokinase receptor-mediated beta3 integrin signaling to cause podocyte injury. J Mol Med (Berl). doi:10.1007/ s00109-012-0960-6

9. Zhang B, Xie S, Shi W, Yang Y (2012) Amiloride off-target effect inhibits podocyte urokinase receptor expression and reduces proteinuria. Nephrol Dial Transplant 27:1746-1755 